\title{
Connective Tissue Structure of the Tree Shrew Optic Nerve and Associated Ageing Changes
}

\author{
Julie Albon, ${ }^{1}$ Sarab Farrant, ${ }^{1}$ Saeed Akbtar, ${ }^{1}$ Rob Young, ${ }^{1}$ Mike E. Boulton, ${ }^{2}$ \\ Gillian Smith, ${ }^{1}$ Martin Taylor, ${ }^{1}$ Jez Guggenbeim, ${ }^{1}$ and James E. Morgan ${ }^{1}$
}

Purpose. To identify the structure and composition of the tree shrew optic nerve to determine its potential as a model for glaucoma.

Methods. Tree shrew optic nerves, aged 4 weeks to 5 years, were wax or cryoembedded for analysis of overall morphology and cellular (glial fibrillary acidic protein [GFAP]) and extracellular matrix (collagen types I, III, IV, V, VI; fibronectin; and elastin) immunolocalization studies. In addition, transmission and scanning electron microscopy were performed. In vivo optic disc imaging was performed by HRT2 and fundus camera photography.

REsults. The optic nerve of the tree shrew comprised regions comparable to the human prelaminar and lamina cribrosa (LC) in the optic nerve head and the retrolaminar region, immediately posterior. The multilayered connective tissue plates of tree shrew LC stretched across the optic nerve canal at the level of the sclera and consisted of collagen types I, III, IV, V, and VI; elastin; and fibronectin. Significant age-related alterations in connective tissue components were indicated. Connective tissue was present in the central retinal vessel sheaths and was identified as longitudinally oriented collagen fibrils in the retrolaminar optic nerve. GFAP immunofluorescence indicated a high concentration of astrocytic processes in the LC. Myelination of axons was evident in the retrolaminar optic nerve. Ultrastructural studies supported the structural organization and spatial distribution of connective tissue.

Conclusions. In contrast to many rodent models of glaucoma, since the tree shrew optic nerve resembles that in humans, especially at the LC, the tree shrew offers an ideal opportunity to investigate glaucoma pathophysiology in a subprimate model. (Invest Opbthalmol Vis Sci. 2007;48:2134-2144) DOI: 10.1167/iovs.06-0084

G laucoma remains a major cause of visual loss world wide. Significant advances have been made in the diagnosis and treatment of the disease, but a greater understanding of the underlying pathophysiology is needed if the progressive damage, which occurs despite apparently effective treatment, is to

From the ${ }^{1}$ Cell and Molecular Biology Unit, School of Optometry and Vision Sciences and CITER (Cardiff Institute of Tissue Engineering and Repair), Cardiff University, Cardiff, Wales, United Kingdom; and the ${ }^{2}$ Department of Ophthalmology and Visual Sciences, University of Texas Medical Branch, Galveston, Texas.

Supported by the National Eye Research Centre, United Kingdom. Submitted for publication January 26, 2006; revised June 21 and October 12, 2006; accepted March 9, 2007.

Disclosure: J. Albon, None; S. Farrant, None; S. Akhtar, None; R. Young, None; M.E. Boulton, None; G. Smith, None; M. Taylor, None; J. Guggenheim, None; J.E. Morgan, None

The publication costs of this article were defrayed in part by page charge payment. This article must therefore be marked "advertisement" in accordance with 18 U.S.C. $\$ 1734$ solely to indicate this fact.

Corresponding author: Julie Albon, Cell and Molecular Biology Unit, School of Optometry and Vision Sciences, Redwood Building, Cardiff University, King Edward VII Avenue, Cardiff, Wales, CF10 4NB UK; albonj@cardiff.ac.uk. be prevented. The use of animal models of the disease has enabled significant advances in our understanding of the events within the optic nerve head that result in retinal ganglion cell death. The lamina cribrosa (LC) has been identified as the structure that is critical in determining axonal degeneration in glaucoma. It is the site at which axonal damage occurs, mediated through the effects of elevated intraocular pressure, through direct compression, mechanical compression of axons, or astrocytic reactivity. ${ }^{1-6}$

Until recently, the primate ${ }^{7}$ has been the most commonly used model of human glaucoma. Although the primate provides excellent homology with the human optic nerve, it is technically and logistically a difficult model and in many laboratories is not a realistic option. Rodent models have become increasingly popular and have provided valuable new insights into the events that lead to axon damage or propagate retinal ganglion cell death. ${ }^{8,9}$ Murine models have also been developed with the advantage that genetic variants can be used to target the role of single molecular pathways in the initiation of axonal damage.

Rodent models have a significant limitation, in that they possess only a rudimentary LC, and this deficit imposes limitations on the extent to which they can be used to investigate the mechanisms by which the LC mediates the effects of variation in the level of IOP. Dogs, cats, and pigs have been proposed as alternatives to the primate. However, although these species have LCs that are better developed than that of the rodent, the development of glaucoma in these animals is not straightforward. In this study, the anatomy of the tree shrew was characterized, with particular reference to the human and age-related changes in the LC. Because age is a major risk factor in glaucoma ${ }^{10}$ and age-related changes in the LC are believed to be important in the increased susceptibility of the elderly to glaucomatous neuropathy, the latter is of particular relevance.

The tree shrew (Tupaia belangeri) is likely to provide a cost-effective alternative to the primate as a glaucoma model that will complement studies performed in rodents. It is a highly developed mammal closely related to the primate. ${ }^{11}$ Thus, there is a high probability that ocular developmental mechanisms are similar to those in humans.

\section{MethodS}

\section{Fundus Imaging of the Tree Shrew}

All experiments adhered to the ARVO Statement for the Use of Animals in Ophthalmic and Vision Research and were conducted in accordance with U.K. Home Office regulations. Two tree shrews, aged 7 months and 5 years, were anesthetized with a cocktail of ketamine $(64 \mathrm{mg} / \mathrm{kg}$ body weight) and xylazine ( $7 \mathrm{mg} / \mathrm{kg}$ body weight). After pupil dilation with $1 \%$ tropicamide (Chauvin Pharmaceuticals, Romford, UK), fundus images were captured with fundus camera (F505; Nikon, Tokyo, Japan) and scanning laser ophthalmoscope (SLO) images were taken with an HRT 2 (Eye Explorer software 1.4, Heidelberg Engineering, Dossenheim, Germany). Fundus camera images were digitized using scanner (Dimage Scan Elite 5400; Minolta, Osaka, Japan) and converted 
to monochrome images (Powerpoint 2003, Microsoft Office; Microsoft, Redmond, WA).

\section{Preparation of Tissue Sections}

Optic nerves of tree shrew, aged 4 weeks to 5 years, were wax $(n=$ 3 ) or cryoembedded $(n=12)$ for analysis of overall morphology and immunolocalization studies. Seven-micrometer wax-embedded and $10-\mu \mathrm{m}$ frozen sections were cut with a microtome and cryostat (Leica, Deerfield, IL), respectively. The frozen sections were air-dried for 30 minutes before storing at $-20^{\circ} \mathrm{C}$.

\section{Hematoxylin and Eosin}

Longitudinal wax-embedded optic nerve sections were brought to water before a 5 minute immersion in Harris' hematoxylin (BDH, Poole, UK). Thereafter, sections were rinsed in running tap water for 10-minutes, counterstained for 2 minutes in eosin, and rinsed again in running tap water. The sections were then dehydrated and mounted in a synthetic resin (DPX) before being imaged with image-capture software (QWin; Leica).

\section{Indirect Immunofluorescence}

Optic nerve cryosections were brought to room temperature for 10 minutes and then immersed in three 10-minute washes in phosphatebuffered saline (PBS; pH 7.4). The sections were then probed, in triplicate, with primary antibodies diluted in PBS or in PBS only (negative control) for 2 hours in a humidified chamber. Primary antibodies (diluted in PBS) included: rabbit anti-human fibronectin (1:100 dilution; Sigma, Poole, UK); goat anti-collagen types I, III, IV or V (1:100 dilution; Southern Biotechnology Inc., Huntsville, AL); rabbit anticollagen type VI (1:50 dilution; Chemicon, Hampshire, UK); rabbit anti-elastin (1:50 dilution; AbD Serotec [formerly Biogenesis], Oxford, UK); and mouse anti-glial fibrillary acidic protein (GFAP, 1:200 dilution; Sigma). Sections probed with anti-GFAP were incubated in $0.2 \%$ TritonX-100 for 10 minutes before antibody incubation. After three 10minute rinses in PBS, sections were then incubated in appropriate secondary AlexaFluor 488 antibodies (Invitrogen-Molecular Probes, Paisley, UK), diluted 1 in 1000 in PBS containing $0.2 \mu \mathrm{g} / \mathrm{mL}$ bisbenzimide for 2 hours, rinsed in three 10-minute changes of PBS, and mounted in gelvatol. The LC and retrolaminar and central retinal vessels were visualized by microscopy. Because the LC is the site of axonal injury in glaucoma, the distribution and level of immunolabeling was evaluated in this region. The LC was graded, from 0 to 4 $(++++)(0$, no staining; 4 , significant immunolabeling throughout the observed structure), according to the distribution and level of immunofluorescence of each antibody. Optic nerve sections were allocated to one of three groups ( $n=4$ optic nerves in each group) according to the age of the tree shrew (group 1, <1 year; group 2, 1-2 years; group 3, 4-5 years) and the images were captured (QFluoro; Leica).

The Fisher exact test was used to analyze the effect of age on immunolocalization grade for each antibody target in the LC of the tree shrew. This test was deemed suitable, as it is designed to assess categorical data in contingency tables, where the sample size is small (i.e., $n \leq 4)$. The null hypothesis, that age had no effect on immunolocalization grade outcome, was rejected if $P<0.05$.

\section{Scanning Electron Microscopy}

Human and tree shrew optic nerves $(n=6)$ were fixed in $2.5 \%$ glutaraldehyde and $2 \%$ paraformaldehyde in $0.1 \mathrm{M}$ sodium cacodylate buffer ( $\mathrm{pH}$ 7.4) for 3 hours before rinsing in buffer at $4^{\circ} \mathrm{C}$ for 24 hours. Optic nerves were longitudinally bisected, immersed in $10 \% \mathrm{NaOH}$ for 36 hours, and rinsed in distilled water for 24 hours. Samples were then postfixed in $1 \%$ osmium tetroxide for 3 hours, washed in distilled water overnight, and dehydrated through a graded ethanol series,
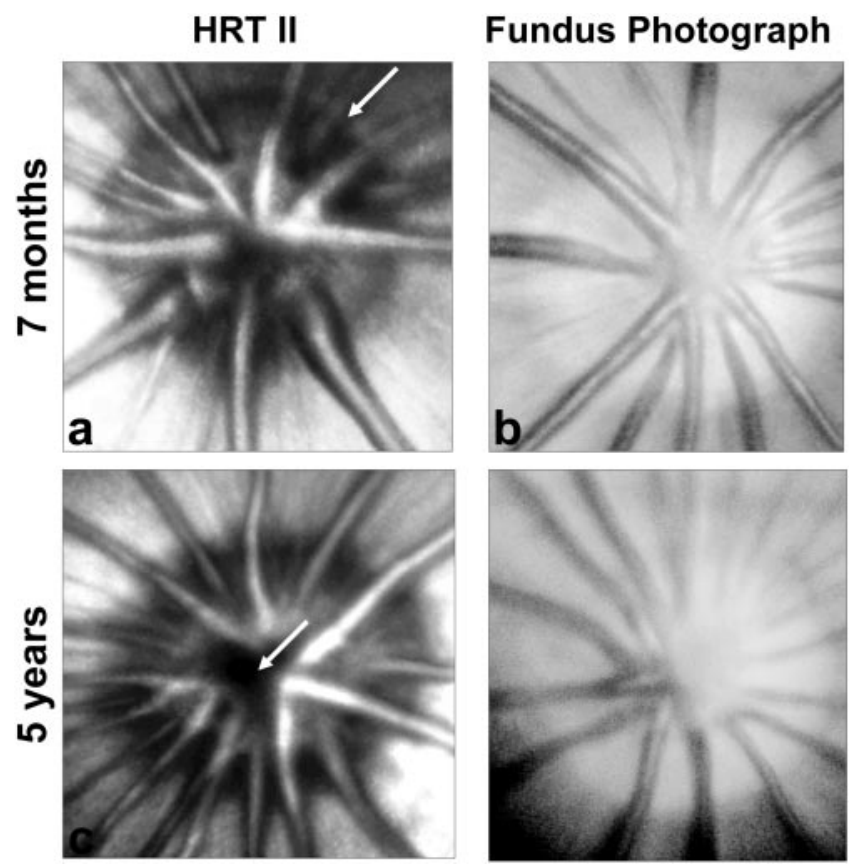

FIGURE 1. In vivo imaging of the tree shrew optic disc by HRTII $(\mathbf{a}, \mathbf{c})$ and fundus photography $(\mathbf{b}, \mathbf{d})$. Images shown are from the right (a) and left (b) eyes of a 7-month-old and the right eye (c, d) of a 5-year-old tree shrew. (a, arrow) Margin of the disc (scleral rim). A small central cup was present in the 5-year-old disc (c, arrow). In all eyes the retinal nerve fiber layer striations can clearly be seen.

including two changes in $100 \%$ ethanol over 3 hours. After criticalpoint drying, the tissue samples were mounted on aluminum stubs with class C cement (Leitz, Wetzlar, Germany), gold-coated with a gold sputter coater (EMscope COOE; Emitech, Ashford, UK) and examined by scanning electron microscope (XL20; Philips, Eindhoven, The Netherlands).

\section{Transmission Electron Microscopy}

Optic nerves were fixed in $2.5 \%$ glutaraldehyde for 2 hours, postfixed in $1 \%$ osmium tetroxide for 1 hour, and washed twice in distilled water. After dehydration through graded ethanols (30\%-100\%, for 15 minutes each), samples were subjected to 1-hour incubations in 1:1 and then 1:2 ethanol-Spurr's resin mixture. After three 8-hour changes in $100 \%$ Spurr's resin, the samples were polymerized at $70^{\circ} \mathrm{C}$ over 8 hours.

Semithin and ultrathin sections were cut (Ultracut microtome; Reichert-Jung, Vienna, Austria). Semithin (1 $\mu \mathrm{m})$ sections were stained with $1 \%$ toluidine blue and visualized by light microscopy. Ultrathin sections were floated onto grids, stained with $2 \%$ uranyl acetate and Reynolds lead citrate, and observed by transmission electron microscopy (model 1010; JEOL, Tokyo, Japan).

\section{RESUlts}

\section{Fundus Imaging}

Representative optic discs of a 7-month-old (Figs. 1a, 1b) and a 5-year-old (Figs. 1c, 1d) male tree shrew are shown in Figure 1. SLO images indicated enlargement of the central cup in the 5-year-old (Fig. 1c) compared with that of the 7-month-old, in which the presence of a cup could not be identified (Fig. 1a). In all eyes, the margin of the optic disc (Elschnig's rim) could easily be discerned, as could the striations of the retinal nerve fiber layer. Retinal vessels were seen to radiate from the center 

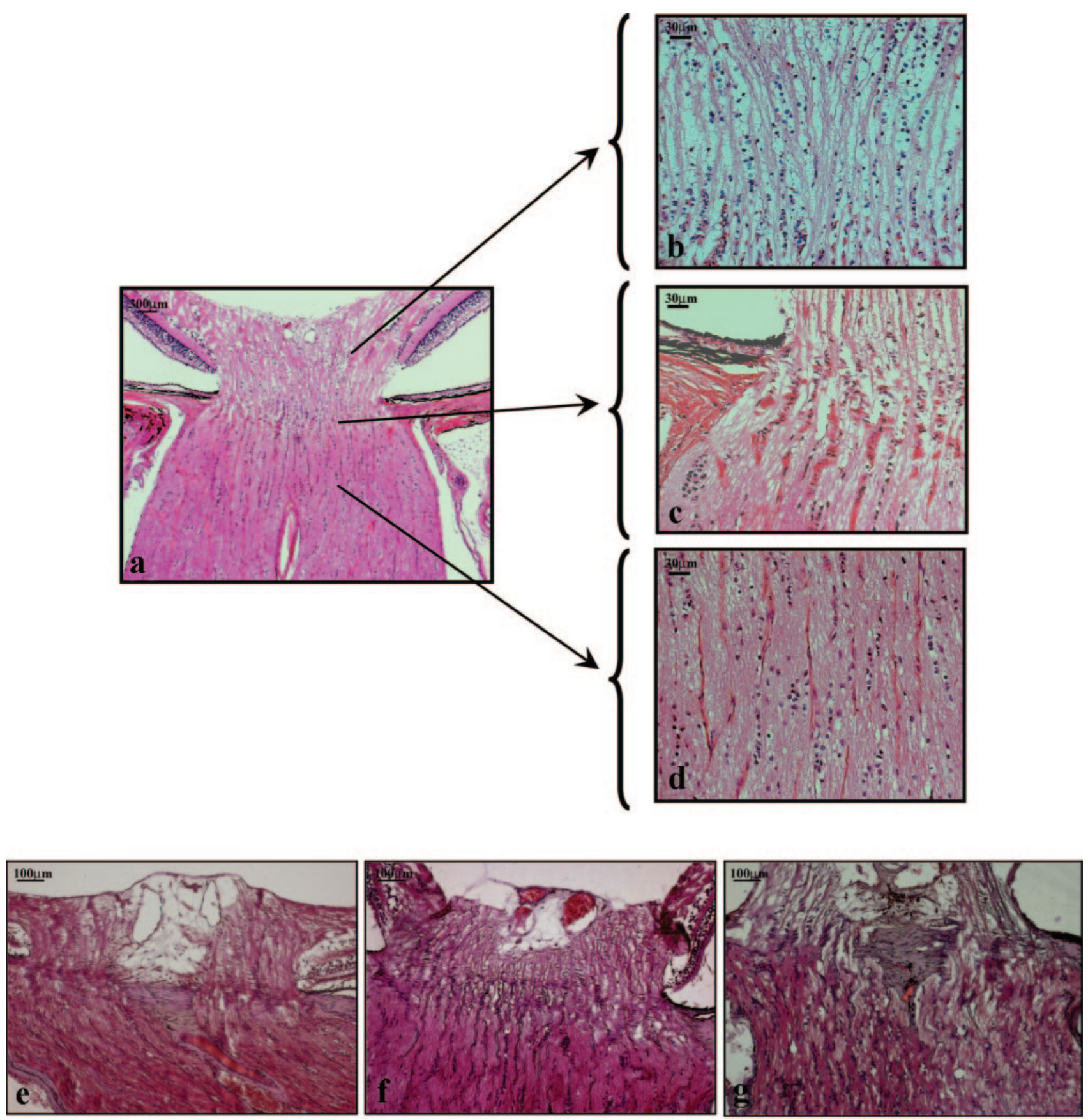

Figure 2. Hematoxylin and eosin-stained tree shrew optic nerve (a). The optic nerve contained structures similar to that in human optic nerve. Retinal ganglion cell axons were segregated into fascicles by cells in the prelaminar region (b). At the level of the sclera, a dense LC-like structure (c) stretched across the optic nerve canal, interspersed between nerve fascicles. Dense connective-like tissue was also evident between axon bundles in the retrolaminar region (d). Representative images for each age group are shown (e, 4 weeks; $\mathbf{f}, 1.75$ years; and $\mathbf{g}, 5$ years).

of the optic disc and were distributed in a radial fashion around the margin of the optic disc.

\section{Overall Morphology: Hematoxylin and Eosin}

The tree shrew optic nerve had three easily discernible compartments (Fig 2). In the region where the retinal ganglion cell axons leave the retina and turn into the optic nerve proper, the axons became segregated into bundles separated by hematoxylin-stained nuclei (Fig. 2b). This region appeared to lack connective tissue and was consistent with the human prelaminar region. However, denser staining, suggestive of a connective tissue structure was identi- fied at the level of the sclera, stretching across the optic nerve canal (Fig. 2c), comparable to the human LC. Below this region, the optic nerve seemed to widen in diameter, and the nerve fiber bundles were still interspersed with connective tissue (Fig. 2d). This latter region was identified as the retrolaminar optic nerve. Representative images of the optic nerve head of the three age groups are shown in Figs. 2e ( 4 weeks), $2 \mathrm{f}$ ( 1.75 years), and $2 \mathrm{~g}$ ( 5 years).

Of interest is that the subarachnoid space appeared to extend up into the immediate peripapillary sclera (Fig. 2a), effectively causing the sclera to be functionally very thin in this region. 


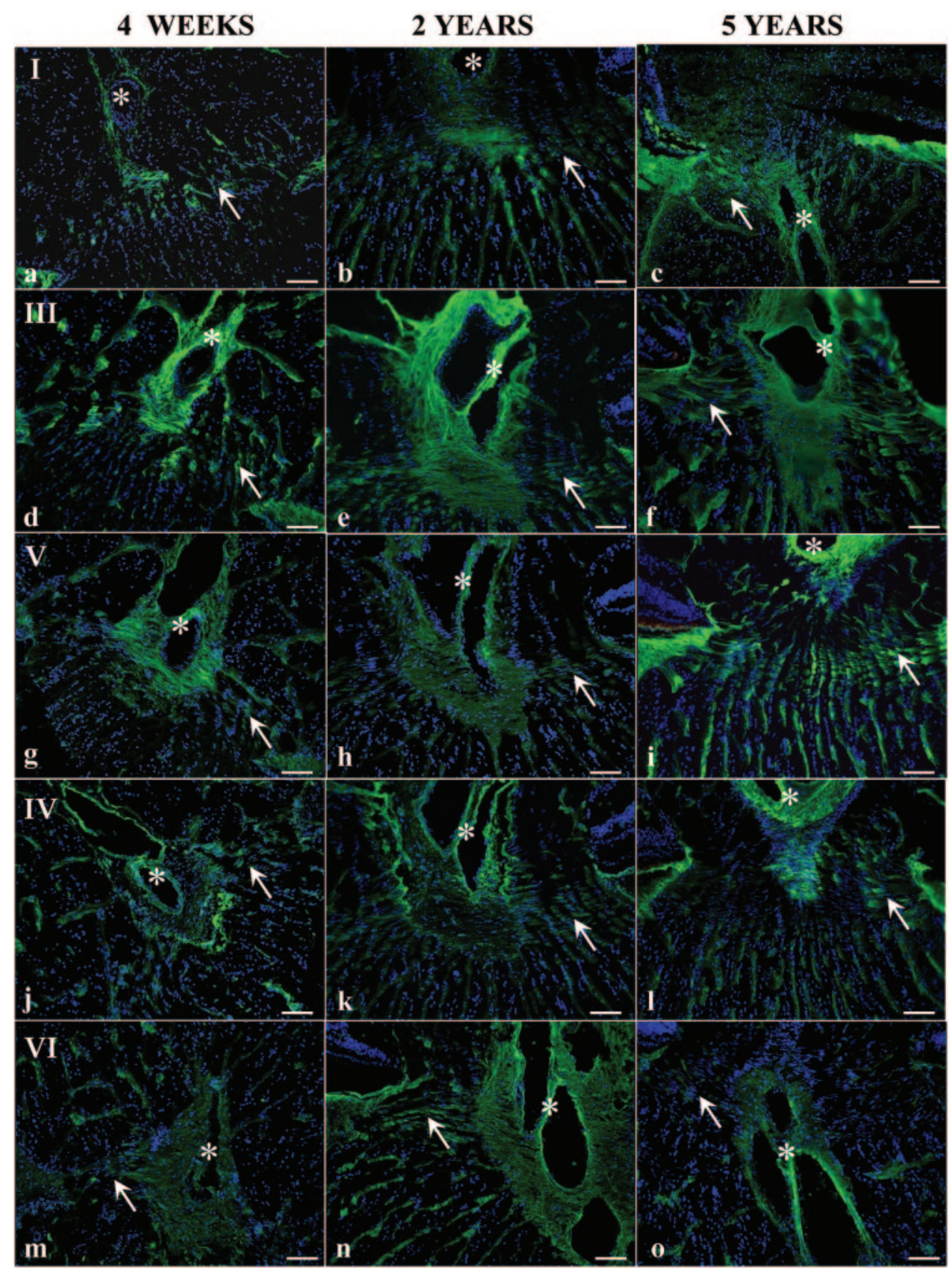

FIGURE 3. Immunofluorescent localization of collagenous components of the extracellular matrix in the tree shrew optic nerve head. Fibrillar collagen types I (a-c), III (d-f), and V (g-i) and nonfibrillar collagens types IV (j-1) and VI (m-o) were present in the multilayered LC-like structure (arrow) and the walls of the central retinal vessels (*) in tree shrews aged 4 weeks to 5 years. Age-related in creases in immunoreactivity for collagen types I (a: 4 weeks, b: 2 years, c: 5 years), III (d: 4 weeks, e: 2 years, $\mathbf{f}: 5$ years), $V$ (g: 4 weeks, h: 2 years, i: 5 years), IV (j: 4 weeks, k: 2 years, $1: 5$ years), and VI (m: 4 weeks, n: 2 years, o: 5 years) were observed. In the central retinal artery and vein, type IV immunoreactivity was limited to the vessel walls. Scale bars, $100 \mu \mathrm{m}$.

\section{Indirect Immunofluorescent Labeling of the Tree Shrew Optic Nerve}

Immunolabeling results in the optic nerve head and a higher magnification of the septal system in the retrolaminar optic nerve are demonstrated in Figures 3 and $4 \mathrm{a}-\mathrm{c}, 4 \mathrm{~g}-\mathrm{i}$, and $4 \mathrm{~m}-\mathrm{o}$, and Figures 5 and $4 \mathrm{~d}-\mathrm{f}, 4 \mathrm{j}-1$, and $4 \mathrm{p}-\mathrm{r}$, respectively. The immunolocalization grading results are tabulated in Table 1 for simplification to show apparent changes in distribution and intensity of immunofluorescence labeling with respect to age. The grade presented in Table 1 is that shown in triplicate sections, since variation in immunofluorescence-related labeling within triplicate sections of a single optic nerve for a particular antibody target was not identified.

\section{Optic Nerve Head}

Positive immunofluorescence labeling was identified for collagen types I, III, IV, V and VI, elastin, fibronectin and GFAP (see
Table 1). Immunofluorescent labeling of the fibrillar collagen types I (Figs. 3a-c), III (Figs. 3d-f), and V (Figs. 3g-i) appeared to be codistributed throughout the connective tissue plates of the tree shrew LC and the walls of the central retinal vessels. Significant changes in immunolabeling for collagen types I (Figs. 3a-c), III (Figs. 3d-f), and V (Figs. 3g-i) were observed with age $(P<0.01)$, with lower levels determined in the 4-week-old LC (Figs. 3a, 3d, 3g) compared with that in the 2-year-old (Figs. 3b, 3e, 3h) and the 5-year-old (Figs. 3c, 3f, 3i) LCs. The network-forming collagens, type IV (Figs. 3j-1) and VI (Figs. $3 \mathrm{~m}-\mathrm{o}$ ) were localized to the cribriform plates of the LC and central retinal vessels. Type IV immunofluorescence, observed in the central retinal artery and vein, was predominantly localized to the borders of the vascular walls. No obvious age-related change in collagen type IV immunolabeling was observed in the vascular walls, although labeling for type IV increased and appeared to be distributed throughout the LC 
4 WEEKS
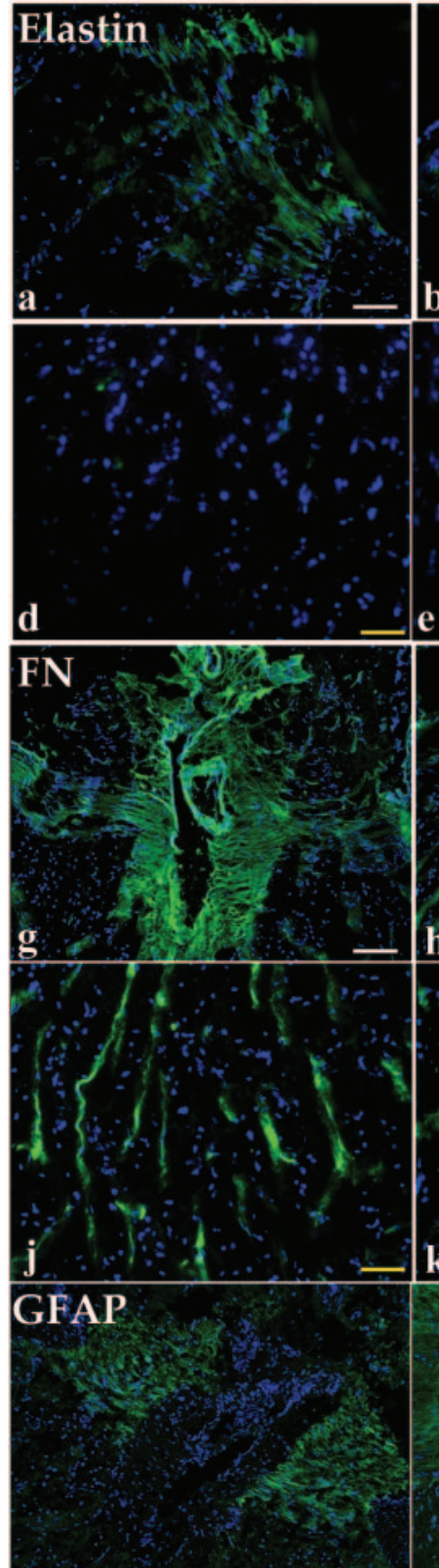

m

n!
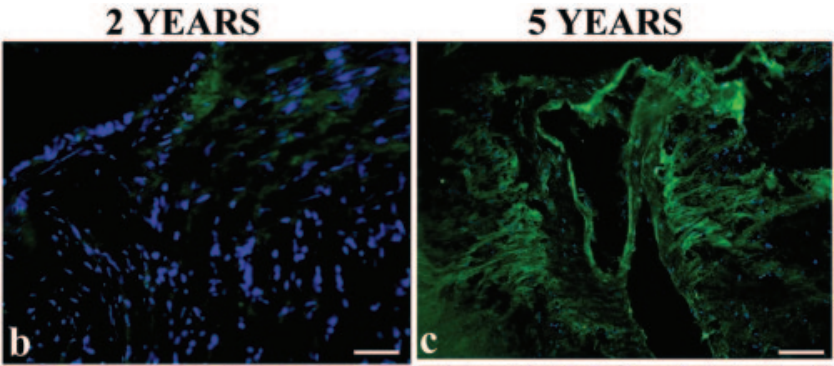

.
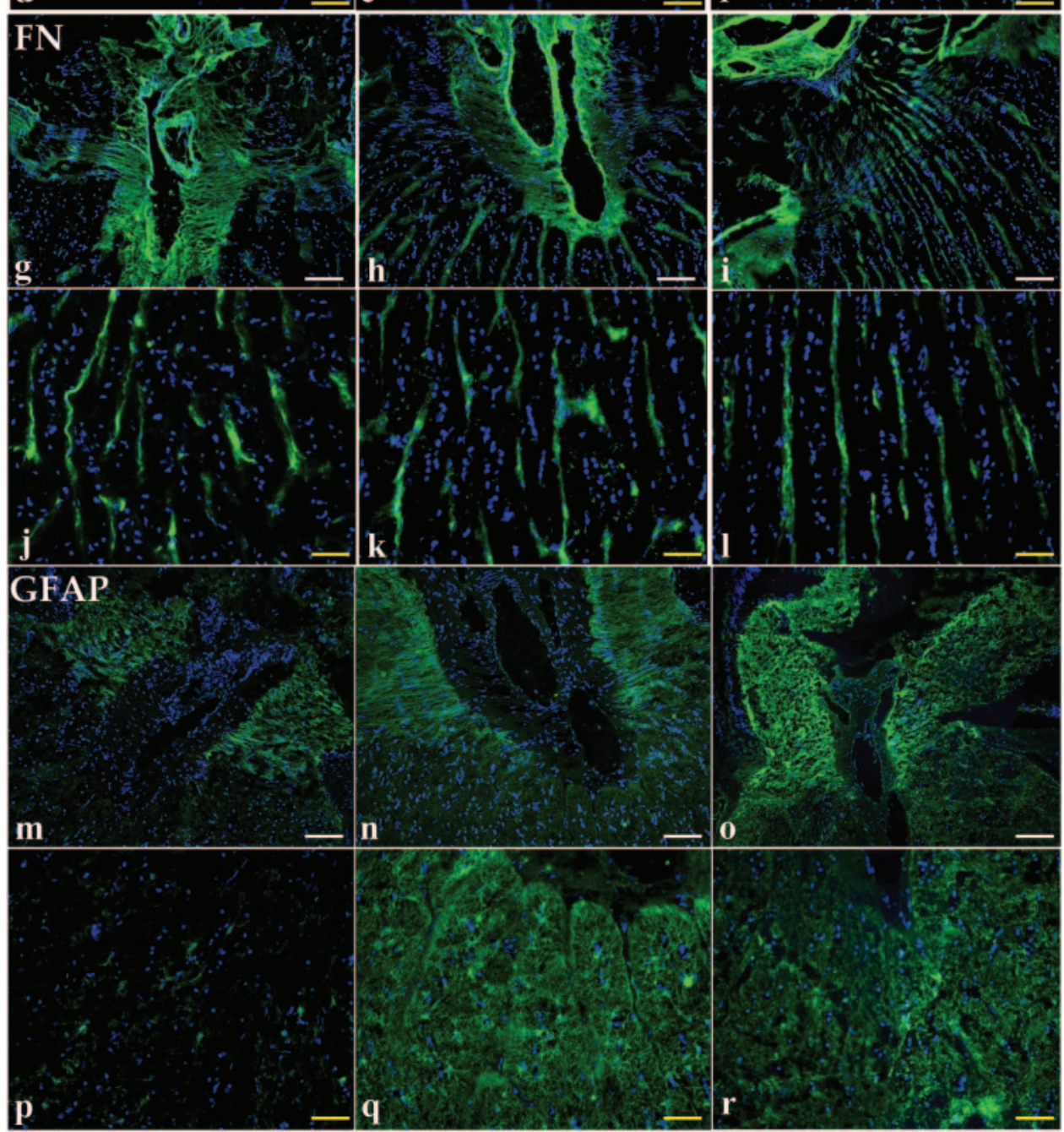
q

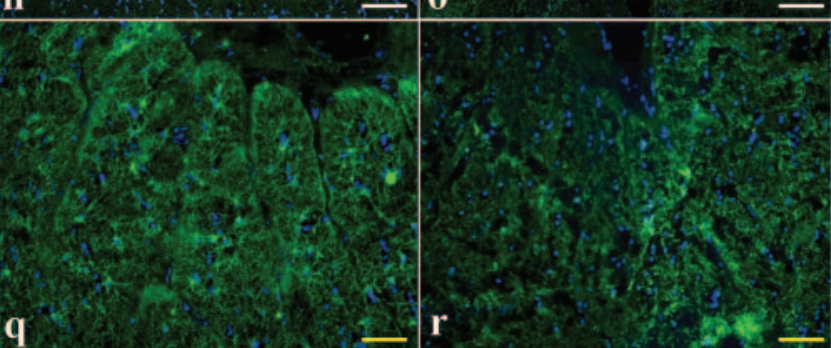

FIGURE 4. Immunofluorescent localization of noncollagenous components in the tree shrew optic nerve head and retrolaminar optic nerve. Images denote the extracellular matrix components elastin (a-f) and fibronectin (g-1) and the astrocyte-specific cytoskeletal marker GFAP (m-r) in different regions of the tree shrew optic nerve, aged 4 weeks to 5 years. An age-related increase in elastin $(\mathbf{a}-\mathbf{c})$ and decrease in fibronectin reactivity was identified in the optic nerve head $(\mathbf{g}-\mathbf{i})$. GFAP immunoreactivity appeared to increase with age and was more intense in the LC (m-o) than in the retrolaminar optic nerve (p-r). Scale bars, (wbite) $100 \mu \mathrm{m}$; (yellow) $40 \mu \mathrm{m}$. with increasing age $(P<0.01)$. Positive labeling for collagen type VI (Figs. 3m-o) demonstrated a similar, but more diffuse, distribution throughout the optic nerve head compared with that of types I, III, and V. As with fibrillar collagen-related labeling, collagen type VI immunofluorescence appeared to increase in distribution throughout the LC with age $(P<0.01$, Figs. 3m-o).
Immunolabeling for elastin (Figs. 4a-c) followed a similar pattern to that of fibrillar collagens within the LC and central retinal artery, with an age-related increase in labeling, such that the laminar beams were intensely labeled in the more elderly tree shrews (Fig. 4c, $P<0.05$ ). Fibronectin-related immunofluorescence appeared to be decrease with age $(P<0.01)$, with intensity of staining in connective tissue of the youngest 
FIGURE 5. Immunofluorescent localization of collagenous components of the extracellular matrix in the tree shrew retrolaminar optic nerve. Fibrillar collagen types I $(\mathbf{a}-\mathbf{c})$, III $(\mathbf{d}-\mathbf{f})$, and $\mathrm{V}(\mathbf{g}-\mathbf{i})$ and nonfibrillar collagens type IV (j-1) and VI (m-o) were present in the connective tissue septae of tree shrews, aged 4 weeks to 5 years. Increased immunoreactivity for collagen types III (f) and V (i) collagen was apparent in the eldest age group ( 5 year old, shown) compared to the younger age groups: 4 weeks (d, $\mathbf{g})$ and 2 years: $(\mathbf{e}, \mathbf{h})$ for types III and V, respectively. Collagen type IV was present both within and along the connective tissue septae in the 2 (k) and (l) 5-year-old septae, with reduced immunoreactivity in the 4-week-old (j). Scale bars, $40 \mu \mathrm{m}$.

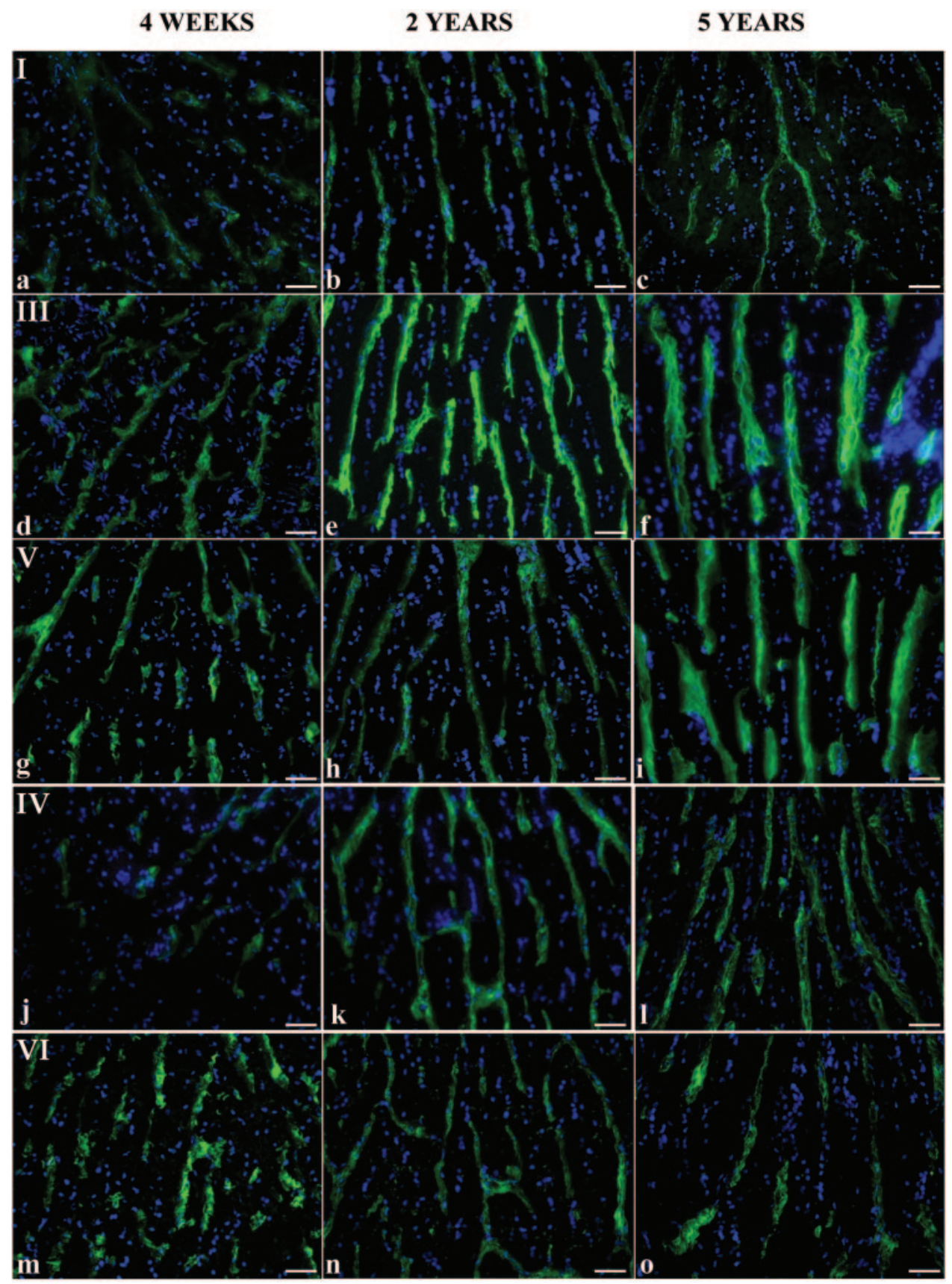

( 4 weeks, Fig. 4g) LC being greater when compared with older tissue (Figs. 4h, 4i).

Intense immunolabeling for GFAP was confined to the LC. Labeling appeared to be oriented in the direction of cell processes extending horizontally across the optic nerve canal (Figs. $4 \mathrm{~m}-\mathrm{O})$, and demonstrated an age-related increase $(P<$ $0.05)$.

It seems likely that there is a border of Elschnig at the periphery of the tree shrew optic nerve, and in some sections, it appeared that fine struts of connective extended upward (Figs. 3c, 3f, 3i, 31).

\section{Retrolaminar Optic Nerve}

Immunofluorescence labeling of the fibrillar collagen types I (Figs. 5a-c), III (Figs. 5d-f), and V (Figs. 5g-i) were codis- tributed throughout the connective tissue of the optic nerve septae. The network-forming collagens types IV (Figs. 5j-1) and VI (Figs. 5m-o) were localized to optic nerve septae.

Immunolabeling for elastin followed a pattern similar to that of fibrillar collagens within the LC, central retinal artery, and retrolaminar optic nerve septae (Figs. 4a-f). Elastin labeling was sparse in the retrolaminar optic nerve septae in optic nerves $<2$ years of age (Figs. $4 \mathrm{~d}, 4 \mathrm{e}$ ), but appeared throughout the septae in the older age group ( 4 to 5 years, Fig. 4f). Fibronectin immunofluorescence was present throughout the optic nerve septae (Figs. 4j-1) in all ages.

GFAP-related immunofluorescence appeared less intense in retrolaminar optic nerve (Figs. 4p-r), with minimal labeling observed in the 4-week-old tree shrew optic nerve (Fig. 4p), compared with labeling throughout the older optic nerve tissue (Figs. 4q, 4r). 
TABLE 1. Distribution of Immunolabeling within the Tree Shrew Optic Nerve

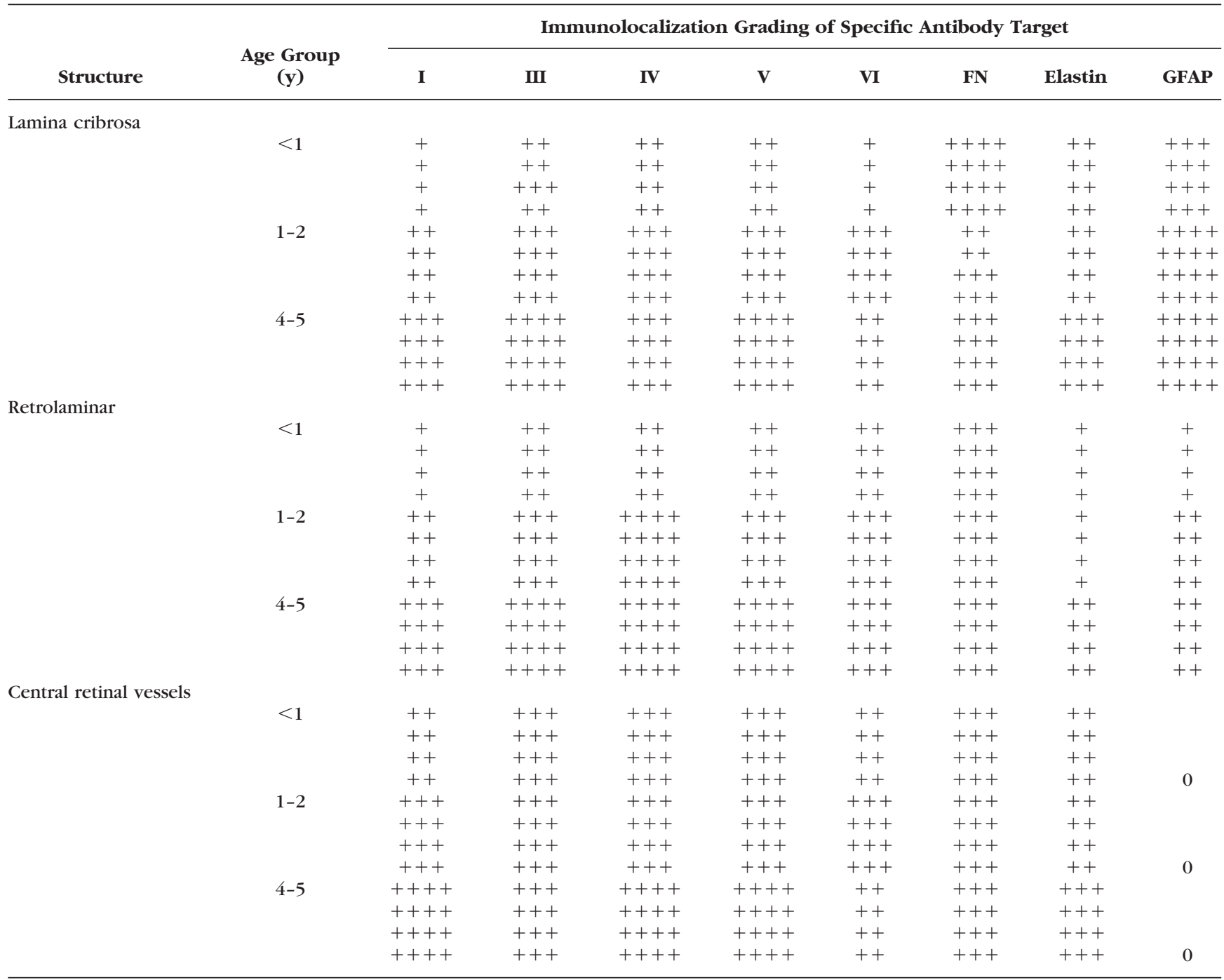

The distribution of labeling for each target antibody is demonstrated in optic nerves allocated to three age groups $(n=4$ optic nerves in each group): $<1,1-2$, and $4-5$ years. Each grade represents immunorelated fluorescence for each target antibody, identified in triplicate sections of each optic nerve within each age group. An age-related increase in collagen types and elastin within cribrosal beams was indicated in the LC. Intense immunolabeling for GFAP was identified in LC at $>1$ year. Intense immunolabeling for fibronectin (FN) appeared in LC at $<1$ year. 0 , no immunostaining; + , minimal immunolocalization; ++ , immunolocalization $>+;+++$, immunolocalization throughout structure; ++++ , immunolocalization $>+++$.

\section{Ultrastructural Analysis of the Tree Shrew Optic Nerve}

At the junction between the LC and the sclera (the insertion region, Fig. 6a), the dense network of closely, compacted collagen was clearly visible. The collagen fibrils were laid down in bundles in a cruciate pattern (Fig. 6a). Collagen fibril bundles in the LC were adjacent to cells (Fig. 6b) and oriented across the optic canal. In contrast, the collagen fibril bundles were observed between optic nerve axons in the retrolaminar region in longitudinal arrays (Figs. 6c). The fibrillar banding pattern of collagen (Figs. 6a-c) in the optic nerve and the myelination of nerve axons in the retrolaminar optic nerve (Fig. 6d) were observed.

\section{Scanning Electron Microscopy: Comparison of Tree Shrew and Human Optic Nerve}

The optic nerve structure of the tree shrew (Figs. 7a-e) was compared to that of the human (Figs. $7 \mathrm{f}-\mathrm{h}$ ), after removal of cellular elements by $\mathrm{NaOH}$ digestion. This allowed the connective tissue architecture of the optic nerves to be visualized by scanning electron microscopy. The optic nerve head of the tree shrew comprised a well-formed LC (Figs. 7a, 7b; Figs. $7 \mathrm{c}-\mathrm{e}, \mathrm{LC}$, asterisk), consisting of several successive connective tissue plates that stretched across the optic nerve canal at the level of the sclera (Figs. 7c-e). In views down through the optic nerve, pores bound by connective tissue were identified in both tree shrew (Fig. 7b) and human (Fig. 7f) LCs. A longitudinal cross-sectional orientation of the optic nerves clearly demonstrated the change in orientation of connective tissue, which is observed as the LC in the optic nerve head (Figs. 7c, $7 \mathrm{~g}, \mathrm{LC}$, asterisk) enters the retrolaminar optic nerve in both tree shrew (Fig. 7c, at higher magnification in Fig. 7e) and human (Fig. 7g). The horizontal arrangement of connective tissue sheets in the LC, perpendicular to the long axis of the optic nerve in the tree shrew (Fig. 7d) is comparable to that in the human LC (Fig. 7h). 
FiguRE 6. Ultrastructural analysis of the tree shrew optic nerve head. (a) Criss-cross arrangements of collagen fibrils at the region of insertion into the sclera. Collagen fibrils were oriented across the optic nerve canal in the LC-like structure (b) and longitudinally in the same direction of nerve axons in postlaminar optic nerve septae (c). Myelin was also identified in the latter region $(\mathbf{d})$.

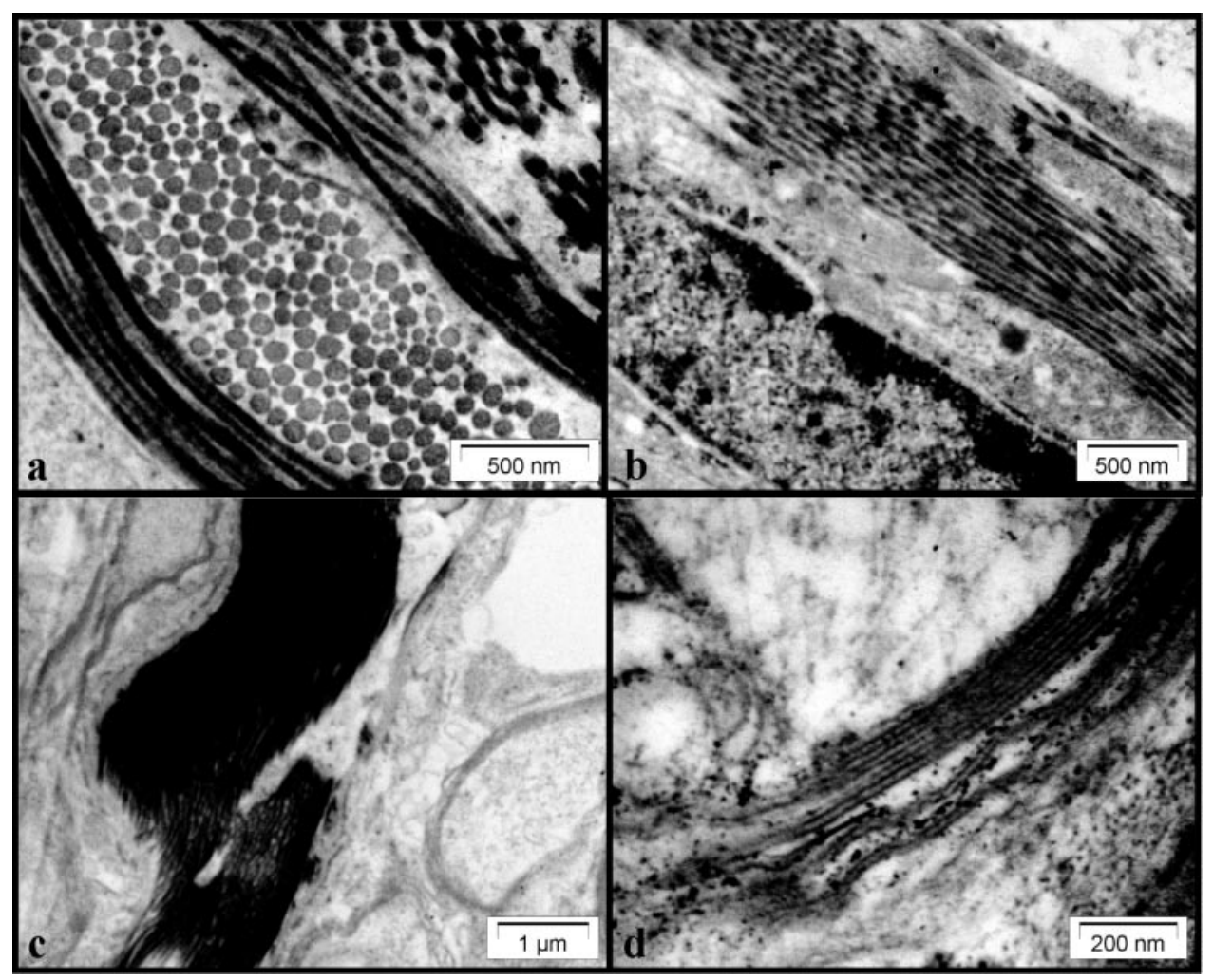

The optic nerve increased in diameter, behind the LC (asterisk) in both the tree shrew (Figs. 7c) and human (Fig. 7g) retrolaminar optic nerves. The connective tissue architecture of the central retinal vasculature is identifiable in a surface view (Fig. 7a) and projecting through and from the LC in the longitudinal view of the tree shrew optic nerve (Fig. 7c). In the human, the central retinal vessels can be seen exiting the optic nerve in Figure $7 \mathrm{~g}$.

\section{Discussion}

Because of similarities in brain, skull, eye development, and reproductive characteristics between tree shrews and primates, the tree shrew family, Tupaiidae, was once classified under the order Primates. However, Tupaiidae are now more commonly designated to the order Scandentia and are characterized as highly developed insectivores and very primitive primates.

The tree shrew optic nerve has many similarities in structure and composition to that of the human, which can be correlated to the human prelaminar and LC in the optic nerve head and retrolaminar optic nerve. The tree shrew optic nerve comprised a noncollagenous region, where the retinal ganglion cell axons are organized into nerve fascicles, which is representative of the human prelaminar. Posterior to this, a connective tissue structure, composed of sheets that stretch horizontally across the optic canal at the level of the sclera, resembled the human LC. As in humans, this LC-like structure is the first form of support to the nerve axons as they pass into the optic nerve. It spanned the scleral canal and appeared to offer support to axon fascicles in a manner similar to that in humans. Collagen fibrils changed their orientation posterior to the LC region. Connective tissue was still evident, but as in the human retrolaminar optic nerve, the collagen fibrils appeared to become longitudinally oriented and the nerve axons were myelinated. Consistent with this, the diameter of the optic nerve increased considerably, compatible with human optic nerve architecture. Because of the many similarities in structure between the tree shrew and human optic nerve, for the purpose of this article, all future references made to optic nerve regions will use the corresponding human nomenclature (i.e., prelaminar, LC, and retrolaminar).

Consistent with studies in human optic nerves, ${ }^{12-17}$ the tree shrew LC and central retinal vessels comprised collagen types I, III, IV, V, and VI. All collagen types were present within the cribriform plates (and also along the edges in the case of type IV collagen) and the septal system that segregates nerve fascicles in the retrolaminar optic nerve. Because age is an important risk factor in glaucoma, alterations in the ageing LC are likely to be significant in susceptibility to axonal damage. The common tree shrew is known to live approximately 2 to 3 years in the wild, with the longest-lived tree shrew in captivity recorded at 12 years. ${ }^{18}$ Both male and female tree shrews reach sexual maturity at 3 months and the female is able to reproduce at 4.5 months. This suggests that the age range examined in this study correlates to a human age range between approximately 5 and 65 to 70 years.

The immunolocalization studies suggested that an age-related increase in overall collagen composition occurs within the tree shrew optic nerve head, consistent with findings identified in humans. ${ }^{12,15,19}$ The fibrillar collagen types I and III, which provide structures with tensile properties, are likely to impart mechanical strength and resilience to the LC. Type V collagen, known to act as an anchoring network and be involved in the regulation of collagen type I fibril diameter, may indirectly influence mechanical stability. Type VI collagen, a flexible connector of cells and collagen fibrils, is likely to affect the mechanical integrity of the LC. The presence of type IV collagen within the cribriform plates is unusual, because type IV collagen distribution is usually associated with basement membranes. However, the distribution of type IV collagen in the tree shrew optic nerve is similar to that described previ- 

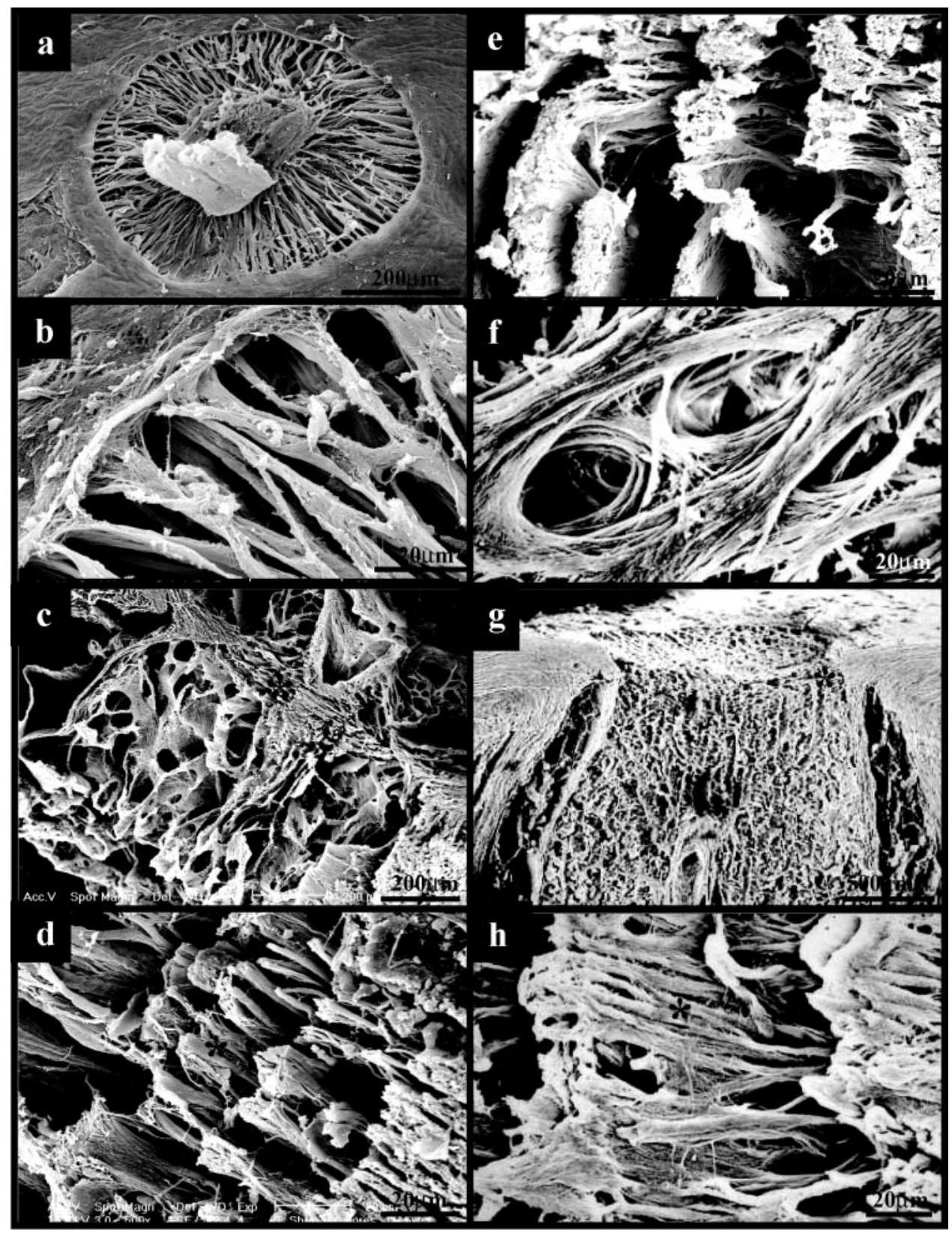

FIGURE 7. Scanning electron micrographs of tree shrew (a-e) and human (f-h) optic nerves. The optic nerve head of the tree shrew comprised a well-formed LC (a, b, denoted by $*$ in $\mathbf{c - e}$ ), consisting of several successive connective tissue plates. Pores bound by connective tissue were identified in both tree shrew (b) and human (f) LCs. Longitudinal cross-sectional orientation of the optic nerves demonstrates the change in orientation of connective tissue, which is observed as the LC (c, $\mathbf{g}$, *) enters the retrolaminar optic nerve in both tree shrew (c, at higher magnification, e) and human (g). The horizontal arrangement of connective tissue sheets in the LC, perpendicular to the long axis of the optic nerve in the tree shrew (d) is comparable to that in the human LC (h). The central retinal vasculature can be identified from a surface view (a) and projecting through and from the LC (*) in the longitudinal view of the tree shrew optic nerve (c) and exiting the human optic nerve (g).

ously in the human optic nerve. ${ }^{12,14,17}$ The presence of elastin within the tree shrew optic nerve head is also compatible with previous observations that elastin in the human optic nerve head $^{20-22}$ is likely to play an important role in its resilience. The disruption of elastic fibers in the LC in glaucoma ${ }^{21,23}$ is, at least in part, likely to contribute to the stiffening of the optic nerve head with progression of glaucoma. ${ }^{24}$

In addition, the intense GFAP immunoreactivity in the optic nerve head region of the tree shrew is consistent with human studies that suggest that the dense meshwork of processes of specialized astrocytes offers structural support to the nerve axons in this region. ${ }^{25,26}$

The distribution and amount of different connective tissue components, as in other connective tissue structures, is vital to the mechanical function and integrity of the LC. Previous studies have shown alterations in these constituents, in both the ageing ${ }^{12,15,19,20}$ and glaucomatous ${ }^{9,27-30}$ LC. Such alterations have been implicated in the changing mechanical prop- erties of the optic nerve head with age $\mathrm{e}^{31}$ and the progression of glaucoma. ${ }^{24,32}$

The similarities between the tree shrew and human LC make it an appealing model in which to study the role of the LC in the initiation of axon damage in glaucoma. The anatomy and structure of the optic nerve head has been studied extensively in the rodent, ${ }^{11,33,34}$ primate,${ }^{30,35}$ and human ${ }^{12-15,17,20,28}$ optic nerve heads. Our study suggests that the tree shrew shows greater homology to human and primate optic nerve than is seen in other models, thus strengthening the case for development of this species as a cost-effective glaucoma model.

Overall, our findings demonstrate that the extracellular matrix composition and structure of the tree shrew optic nerve head have a close correlation and many similarities to that of the human optic nerve head. The tree shrew LC, as in humans, ${ }^{36}$ is inserted into the scleral region by interconnected collagen fibrils, and is composed of successive connective tissue plates that stretch horizontally across the optic canal. 
The thinning in the area of the tree shrew peripapillary sclera in normal eyes suggests that the effective thickness of the peripapillary sclera may be less than would be measured, thus perhaps making the eye more susceptible to deformation and injury. The tree shrew LC contrasts with the poorly developed LC structure reported in rodents. Although the rodent and mouse have been used with great success to study glaucoma, in the rat optic nerve head, the connective tissue plates are longitudinally oriented in the rodent ${ }^{33}$ and the mouse lacks an LC. ${ }^{34}$

Age and elevated IOP are major risk factors for glaucoma, and it is likely that ageing changes in mechanical properties of the $\mathrm{LC}^{31}$ make the elderly more susceptible to glaucoma. The resemblance in structure of the tree shrew optic nerve to that in the human, together with ageing changes in extracellular matrix, illustrates its potential in understanding the cause and/or effect of changes in structure of the LC on cellular responses and axonal loss in both ageing and glaucomatous optic neuropathy.

The tree shrew has been used in several studies of the visual system and is a classic model for myopia. It is possible that the interaction of myopia and the development of glaucoma could be studied in this model-in particular, the possibility that remodeling of sclera around the optic nerve could compromise the structural integrity of the LC and predispose to axon damage. ${ }^{37}$ In addition, other ocular anatomic features may support the advent of the tree shrew as a model to study glaucoma. The tree shrew retina contains three classes of retinal ganglion cell axons RGCs, ${ }^{38}$ that correspond functionally to classes $\mathrm{W}, \mathrm{X}$, and $\mathrm{Y}$ in the cat retina. The RGCs can be categorized according to size of their axons-small, medium, and large-which appear to have an ordered spatial distribution within the optic nerve. Tree shrew RGC axons, as in humans, ${ }^{39}$ also appear to decrease in diameter with age. At this time, the morphology of RGCs in the tree shrew retina is unknown. However, based on the distribution of axon sizes, it is likely that these will follow the distribution seen in the primate.

In summary, the well-developed LC and optic nerve head of the tree shrew suggests that it would be a suitable subprimate model for the study of the effects of elevated IOP on the development of retinal ganglion cell death in experimental glaucoma. Included in this are determination of the age-related changes in structure and resultant biomechanical alterations of the optic nerve head and the effect of such changes in susceptibility to the development, and thereafter progression, of glaucomatous optic neuropathy.

Further characterization of the tree shrew is needed to ascertain its full potential as a model for glaucoma. This investigation should include examination and characterization of the tree shrew optic nerve head vasculature, including the presence of capillaries within the laminar beams and their relationship to cribrosal cells.

\section{Acknowledgments}

The authors thank Cameron Hudson and Michelle Brookes for their expertise in HRTII and fundus photography, respectively.

\section{References}

1. Minckler DS, Bunt AH, Johanson GW. Orthograde and retrograde axoplasmic transport during acute ocular hypertension in the monkey. Invest Opbthalmol Vis Sci. 1977;16:426-441.

2. Quigley HA, Anderson DR. Distribution of axonal transport blockade by acute intraocular pressure elevation in the primate optic nerve head. Invest Ophthalmol Vis Sci. 1977;16:640-644.

3. Quigley HA, Addicks EM. Chronic experimental glaucoma in primates. II. Effect of extended intraocular pressure elevation on optic nerve head and axonal transport. Invest Ophthalmol Vis Sci. 1980;19:137-152.

4. Quigley HA, Addicks EM, Green WR, Maumenee AE. Optic nerve damage in human glaucoma. II. The site of injury and susceptibility to damage. Arch Opbthalmol. 1981;99:635-649.

5. Bellezza AJ, Rintalan CJ, Thompson HW, Downs JC, Hart RT, Burgoyne CF. Deformation of the lamina cribrosa and anterior scleral canal wall in early experimental glaucoma. Invest Ophthalmol Vis Sci. 2003;44:623-637.

6. Quigley HA, Hohman RM, Addicks EM, Massof RW, Green WR. Morphologic changes in the lamina cribrosa correlated with neural loss in open-angle glaucoma. Am J Ophthalmol. 1983;95:673-691.

7. Quigley HA, Dorman-Pease ME, Brown AE. Quantitative study of collagen and elastin of the optic nerve head and sclera in human and experimental monkey glaucoma. Curr Eye Res. 1991;10:877888 .

8. Johnson EC, Morrison JC, Farrell S, Deppmeier L, Moore CG, McGinty MR. The effect of chronically elevated intraocular pressure on the rat optic nerve head extracellular matrix. Exp Eye Res. 1996;62:663-674.

9. Morrison JC, Johnson EC, Cepurna W, Jia L. Understanding mechanisms of pressure-induced optic nerve damage. Prog Retin Eye Res. 2005;24:217-240.

10. Quigley HA. Number of people with glaucoma worldwide. $B r J$ Opbthalmol. 1996;80:389-393.

11. Campbell CB. Taxonomic status of tree shrews. Science. 1996;153: 436.

12. Albon J, Karwatowski WS, Avery N, Easty DL, Duance VC. Changes in the collagenous matrix of the aging human lamina cribrosa. BrJ Opbthalmol. 1995;79:368-375.

13. Hernandez MR, Igoe F, Neufeld AH. Extracellular matrix of the human optic nerve head. Am J Ophthalmol. 1986;102:139-148.

14. Hernandez MR, Luo XX, Igoe F, Neufeld AH. Extracellular matrix of the human lamina cribrosa. Am J Opbthalmol. 1987;104:567576

15. Morrison JC, Jerdan JA, Dorman ME, Quigley HA. Structural proteins of the neonatal and adult lamina cribrosa. Arch Opbthalmol. 1989; $107: 1220-1224$.

16. Morrison JC, L'Hernault NL, Jerdan JA, Quigley HA. Ultrastructural location of extracellular matrix components in the optic nerve head. Arch Opbthalmol. 1989;107:123-129.

17. Goldbaum MH, Jeng SY, Logemann R, Weinreb RN. The extracellular matrix of the human optic nerve. Arch Opbthalmol. 1989; 107:1225-1231.

18. Jones M. Longevity of captive mammals. Zool Gart. 1982;52:113128.

19. Hernandez MR, Luo XX, Andrzejewska W, Neufeld AH. Age-related changes in the extracellular matrix of the human optic nerve head. Am J Opbthalmol. 1989;107:476-484.

20. Albon J, Karwatowski WS, Easty DL, Sims TJ, Duance VC. Age related changes in the non-collagenous components of the extracellular matrix of the human lamina cribrosa. Br J Opbthalmol. 2000;84:311-317.

21. Hernandez MR. Ultrastructural immunocytochemical analysis of elastin in the human lamina cribrosa: changes in elastic fibers in primary open-angle glaucoma. Invest Opbthalmol Vis Sci. 1992; 33:2891-2903.

22. Quigley EN, Quigley HA, Pease ME, Kerrigan LA. Quantitative studies of elastin in the optic nerve heads of persons with primary open-angle glaucoma. Opbthalmology. 1996;103:1680-1685.

23. Quigley H, Pease ME, Thibault D. Change in the appearance of elastin in the lamina cribrosa of glaucomatous optic nerve heads. Graefes Arch Clin Exp Ophthalmol. 1994;232:257-261.

24. Zeimer RC, Ogura Y. The relation between glaucomatous damage and optic nerve head mechanical compliance. Arch Opbthalmol. 1989;107:1232-1234.

25. Anderson DR. Ultrastructure of human and monkey lamina cribrosa and optic nerve head. Arch Opbthalmol. 1969;82:800 - 814.

26. Elkington AR, Inman CB, Steart PV, Weller RO. The structure of the lamina cribrosa of the human eye: an immunocytochemical and electron microscopical study. Eye. 1990;4:42-57. 
27. Hernandez MR, Andrzejewska WM, Neufeld AH. Changes in the extracellular matrix of the human optic nerve head in primary open-angle glaucoma. Am J Ophthalmol. 1990;109:180-188.

28. Hernandez MR, Ye H. Glaucoma: changes in extracellular matrix in the optic nerve head. Ann Med. 1993;25:309-315.

29. Quigley HA, Brown A, Dorman-Pease ME. Alterations in elastin of the optic nerve head in human and experimental glaucoma. $\mathrm{BrJ}$ Ophthalmol. 1991;75:552-557.

30. Morrison JC, Dorman-Pease ME, Dunkelberger GR, Quigley HA. Optic nerve head extracellular matrix in primary optic atrophy and experimental glaucoma. Arch Ophthalmol. 1990;108:1020-1024.

31. Albon J, Purslow PP, Karwatowski WS, Easty DL. Age related compliance of the lamina cribrosa in human eyes. Br J Ophthalmol. 2000;84:318-323.

32. Burgoyne CF, Quigley HA, Thompson HW, Vitale S, Varma R. Early changes in optic disc compliance and surface position in experimental glaucoma. Ophthalmology. 1995;102:1800-1809.

33. Morrison J, Farrell S, Johnson E, Deppmeier L, Moore CG, Grossmann E. Structure and composition of the rodent lamina cribrosa. Exp Eye Res. 1995;60:127-135.
34. May CA, Lütjen-Drecoll E. Morphology of the murine optic nerve. Invest Ophthalmol Vis Sci. 2002;43:2206-2212.

35. Morrison JC, Jerdan JA, L'Hernault NL, Quigley HA. The extracellular matrix composition of the monkey optic nerve head. Invest Ophthalmol Vis Sci. 1988;29:1141-1150.

36. Thale A, Tillmann B, Rochels R. SEM studies of the collagen architecture of the human lamina cribrosa: normal and pathological findings. Ophthalmologica. 1996;210:142-147.

37. Burgoyne CF, Downs JC, Bellezza AJ, Suh JK, Hart RT. The optic nerve head as a biomechanical structure: a new paradigm for understanding the role of IOP-related stress and strain in the pathophysiology of glaucomatous optic nerve head damage. Prog Retin Eye Res. 2005;34:39-73.

38. Drenhaus U, von Gunten A, Rager G. Classes of axons and their distribution in the optic nerve of the tree shrew (Tupaia belangeri). Anat Rec. 1997;249:103-116.

39. Repka MX, Quigley HA. The effect of age on normal human optic nerve fiber number and diameter. Ophthalmology. 1989;96:2632. 